\title{
Steady and unsteady RANS simulations of pollutant dispersion around isolated cubical buildings: Effect of large-scale fluctuations on the concentration field
}

\author{
Yoshihide Tominaga ${ }^{\mathrm{a}, *}$, Ted Stathopoulos ${ }^{\mathrm{b}}$ \\ 5 a Niigata Institute of Technology, Kashiwazaki, Niigata, Japan \\ b Concordia University, Montreal, Quebec, Canada
}

\section{A R T I C L E I N F O}

\section{Article history:}

Received 1 November 2016

Received in revised form

22 January 2017

Accepted 2 February 2017

\section{Keywords:}

CFD

Unsteady RANS

Near-field dispersion

Cubical building

\begin{abstract}
A B S T R A C T
The performance of unsteady Reynolds-Averaged Navier-Stokes equations (URANS) for simulations of flow and dispersion fields around isolated cubical buildings has been examined in this study. URANS results were compared with those obtained from steady-RANS (SRANS) computations and experiments. The comparison determines not only the applicability of URANS simulations, but also the contribution of unsteady large-scale fluctuations to pollutant dispersion around buildings. Three different source locations, i.e. upwind, rooftop and downwind releases, were considered for pollutant dispersion around the building. It was found that the improvement of the predicted concentration field achieved by URANS largely depends on the source location. Although this improvement was not as significant in the upwind and rooftop release cases, the prediction accuracy achieved by URANS was substantially improved for the downwind release case, for which, the unsteady-RANS simulations yielded larger estimates of the momentum and concentration diffusions behind the building than SRANS did, improving the accuracy of the estimation of the mean concentration.
\end{abstract}

(c) 2017 Elsevier Ltd. All rights reserved.

\section{Introduction}

Air pollution near and around buildings is an important environmental problem. However, it is difficult to predict pollutant dispersions with certainty because of the complexity of the interaction between atmospheric flow and flow around buildings. A micro-scale computational fluid dynamics (CFD) technique has been used as a powerful tool for investigating such complex interactions, as reviewed in several studies (Li et al., 2006; Blocken et al., 2011; Tominaga and Stathopoulos, 2013; Di Sabatino et al., 2013; Blocken, 2014; Lateb et al., 2016; Tominaga and Stathopoulos, 2016). The CFD technique has several advantages, for instance, it can provide the entire flow and dispersion field data, without suffering from similarity requirements.

The pollutant dispersion process is highly influenced by large and complex velocity fluctuations generated around buildings. These fluctuations occur over a wide range of both temporal and spatial scales. Except for changes in the atmospheric boundary layer itself, the velocity fluctuations around buildings consist of

\footnotetext{
* Corresponding author.

E-mail addresses: tominaga@abe.niit.ac.jp (Y. Tominaga), statho@bcee.concordia.ca (T. Stathopoulos).
}

large-scale organized motion and small-scale turbulence. The large-scale organized motion around buildings is represented by non-stationary flows, such as periodic or quasi-periodic flows involving deterministic patterns. The turbulence can be modeled by RANS (Reynolds-Averaged Navier-Stokes) equations but the largescale motion cannot be reproduced by steady-RANS (SRANS) computation. Numerous studies have compared steady-RANS and unsteady computational methods, such as Large Eddy Simulation (LES), for dispersion modeling around buildings (Stevens et al., 2000; Xie and Castro, 2009; Dejoan et al., 2010; Santiago et al., 2010; Tominaga and Stathopoulos, 2010, 2011, 2012; Salim et al., 2011; Gousseau et al., 2011a,b; Yoshie et al., 2011). LES has been shown to yield better results than RANS for concentration distributions, because the horizontal and vertical diffusions of concentration are reproduced well by LES. The improvement can be mainly attributed to the fact that LES is able to capture the physics of separated regions, and produces time-dependent and threedimensional flow information, such as the Reynolds stresses, that cannot be extracted from RANS (Murakami et al., 1992; Rodi, 1997; Bechmann, 2006; Tominaga et al., 2008a). However, because turbulence modeling is completely different in RANS and LES, it is very difficult to isolate the influence of large-scale flow unsteadiness on the predicted dispersion field. Therefore, unsteady-RANS 


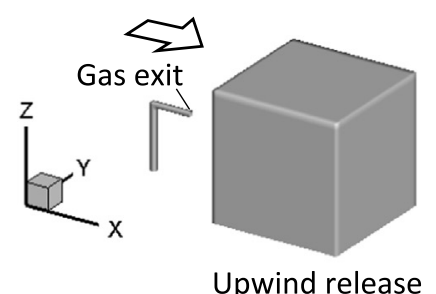

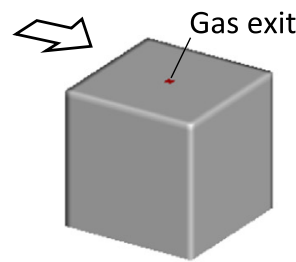

Rooftop release

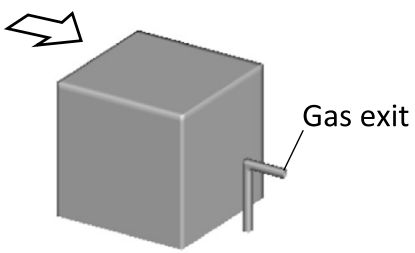

Downwind release

Fig. 1. Schematics of cases analyzed.

(URANS) computations should be viewed as potentially valuable in clarifying this influence.

In previous research, URANS simulations have been successfully used for flows with deterministic unsteadiness, such as vortex shedding in the wake of a two-dimensional obstacle with a low-turbulence approach flow (Kato and Launder, 1993; Rodi, 1993; Murakami and Mochida, 1995; Shimada and Ishihara, 2002; Ramesh et al., 2006; Mannini et al., 2010a, 2010b; Pellegrino and Meskell, 2013). However, only few examples exist of the application of URANS to flows around three-dimensional bluff bodies exposed to relatively high-turbulence approach flows, such as atmospheric boundary layer flows, which are common in wind engineering. Such flows involve complex interactions between largescale organized unsteady flow and random turbulence motions. In an early application of URANS to a flow around a three-dimensional bluff body, Iaccarino et al. (2003) demonstrated that a URANS simulation based on the $v^{2}-f$ turbulence model (Durbin, 1995) could predict periodic shedding and could also produce much better agreement with experimental data than that obtained by SRANS computation for a flow over a wall-mounted cube. Isaev and Lysenko (2009) also found that URANS adequately predicted the characteristics of periodic turbulent separated flows for the same flow configuration as shown by Iaccarino et al. (2003). Shao et al. (2012) used three non-linear $k-\varepsilon$ models to predict flow around an isolated high-rise building with unsteady computations. The study reported that the non-linear models could reproduce the periodic fluctuations and improve the prediction accuracy of the recirculation flow behind the building. Recently, Tominaga (2015) confirmed that URANS computations could reproduce large-scale fluctuations around a high-rise building model and improve the prediction accuracy of the flow behind the building. Because URANS simulation significantly enhances the reproduction of the mixing effect behind a building, it is expected to be particularly effective in the prediction of pollutant dispersions around buildings, as noted by Tominaga and Stathopoulos (2013). However, almost no examples exist of the application of URANS to near-field dispersion around buildings.

In this study, the performance of URANS in simulating flow and pollutant dispersion fields around isolated cubical buildings with different source locations was examined by comparing the results with those obtained by SRANS computation and experiments. This aims to clarify not only the applicability of URANS, but also the influence of large-scale flow unsteadiness on the predicted dispersion field, by investigating the difference between the results of SRANS and URANS. Such an experiment can be done only by URANS, in which the large-scale flow unsteadiness can be isolated. The impact of the source locations on the difference is also discussed regarding unsteady flow behaviors around the building.

\section{Description of wind tunnel experiments}

The experimental data compared here is a part of the database provided for use in validating CFD simulations by the Research Institute for Environmental Management Technology, the National
Institute of Advanced Industrial Science and Technology (AIST) (Iizuka et al., 2008; AIST, 2013). This database was constructed by a study group of CFD applied to atmospheric environmental assessment on behalf of the Japan Society for Atmospheric Environment (Kondo et al., 2009; Mizuno, 2013; Meroney et al., 2016). In this experiment the concentrations on the ground floor were measured precisely, because the intent was to evaluate the applicability of different models to the environmental impact assessment.

\subsection{Building configurations}

An isolated cubical building was adopted as the basic building configuration. Although the experiments were conducted for eight different source locations in total, the typical three locations were selected for evaluation in this study. A schematic of the three cases is shown in Fig. 1.

\subsection{Experimental settings}

The experiments were carried out in the closed-circuit atmospheric boundary layer wind tunnel at AIST, which has a test section of $20 \mathrm{~m}$ length and a cross-section of $3.0 \times 2.0 \mathrm{~m}^{2}$. Vortex generators with height of $0.4 \mathrm{~m}$ were used to create an approachflow wind profile representative of the lower part of a neutral atmospheric boundary layer. The mean streamwise velocity of this incident flow follows the 1/7 power law. Fig. 2 shows the incident vertical profiles of the dimensionless mean velocity $U / U_{H}\left(U_{H}\right.$ : the inflow velocity at the building height $H$ ) and turbulent kinetic energy $k / U_{H^{2}}$, i.e. those measured at a location $11.7 \mathrm{~m}$ downstream from the inlet of the wind tunnel, which is the origin of all measurements. Please note that the profiles were confirmed to be almost identical at further downstream locations. The building Reynolds number, which was determined by $H(0.1 \mathrm{~m})$ and $U_{\mathrm{H}}$ $(1.7 \mathrm{~m} / \mathrm{s})$, was $1.2 \times 10^{4}$. The aerodynamic roughness length $z_{0}$, deduced from the line fitted to the mean velocity profile, was $0.002 \mathrm{~m}$. Applying this $\mathrm{z}_{0}$ value to the logarithmic law, the friction velocity $u^{*}$ for the experimental conditions can be calculated to be approximately equal to $0.2 \mathrm{~m} / \mathrm{s}$. The roughness Reynolds number
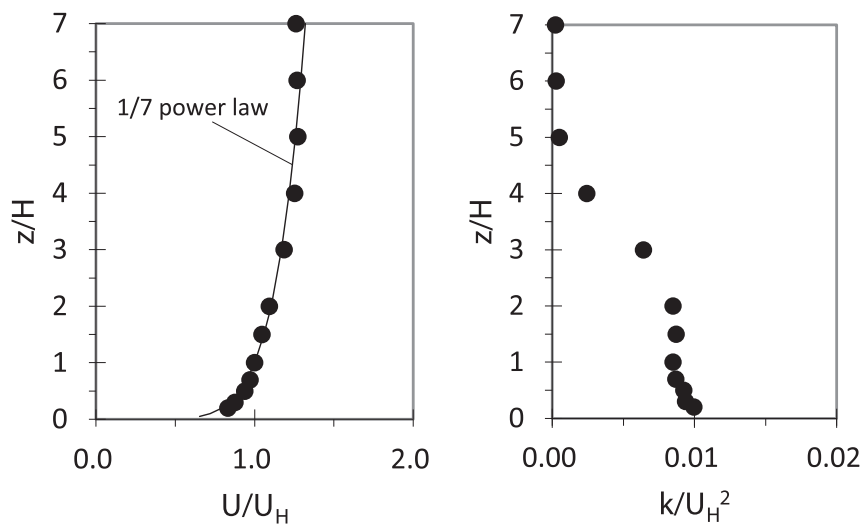

Fig. 2. Incident profiles of mean velocity $U$ and turbulent kinetic energy $k$. 


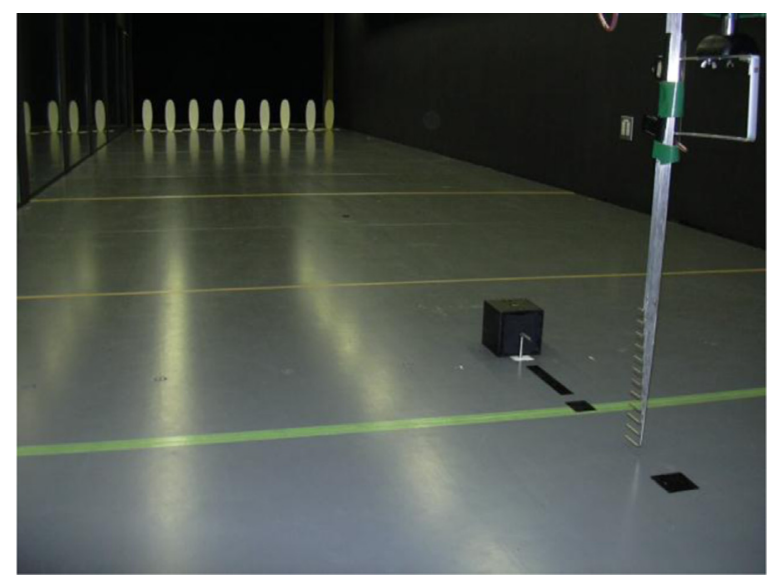

Fig. 3. Experimental setup for the concentration measurements (courtesy of Dr. H. Kondo, National Institute of Advanced Industrial Science and Technology).

based on these values of $z_{0}$ and $u^{*}$ was approximately 23 . Therefore, these experimental conditions satisfy the criteria for a fully rough surface (Snyder and Castro, 2002). The gas exit was modeled as a square of area equal to $1.3 \times 10^{-5} \mathrm{~m}^{2}$ for the upwind case and $2.8 \times 10^{-5} \mathrm{~m}^{2}$ for the rooftop and downwind cases. A concentration of $10 \%$ ethane was emitted at $813 \mathrm{cc} / \mathrm{min}$ for the upwind release case and $400 \mathrm{cc} / \mathrm{min}$ for the rooftop and downwind release cases. These emission rates correspond to momentum ratios (M) of 0.65 and 0.14 , respectively. $\mathrm{M}$ is defined as the ratio of $V_{e} / U_{H}$, where $V_{e}$ is the exhaust velocity.

\subsection{Measurements}

The wind velocity was measured using a laser Doppler anemometer (Dantec Dynamics, Fiber flow, BSA F60 Flow Processor), and the concentration was measured using a carbon-hydrogen analyzer (HADA-01, Kimoto Electric). Fig. 3 shows the experimental setup for the concentration measurements. The time averaging was conducted for periods of $240 \mathrm{~s}$ to obtain the statistical values with a sampling rate of $200 \mathrm{~Hz}$ for the velocity, and $120 \mathrm{~s}$ with $1 \mathrm{~Hz}$ for the concentration. The mean and standard deviation of the three velocity components were measured in the vertical mid-plane $(y / H=0)$ and the horizontal plane at $z=0.5 \mathrm{H}$. Mean concentrations were also measured in the vertical midplane and the horizontal plane on the ground $(z / H=0)$. Fig. 4 shows the locations of the measurement points in each plane for the rooftop release case. Although the locations of the measurement points were slightly different for the other two cases, their resolutions were almost identical.

\section{Description of CFD simulations}

\subsection{Computational geometry and grid}

The computational domain encompassed a volume of $16 H(x) \times$ $12 H(y) \times 7 H(z)$, which was discretized into approximately 0.6 million hexahedral cells for the rooftop release case and 1.2 million hexahedral cells for the upwind and downwind release cases. The computational domain and boundary conditions are shown in Fig. 5(a). The applied grid on the building and ground surfaces for the downwind release case are also shown in Fig. 5(b). A large number of grids was necessary to reproduce the stacks for the upwind and downwind release cases. The building width and height were divided into approximately 20 cells each for all cases. In this study, a grid-sensitivity analysis was performed based on two additional grids: a coarser grid and a finer grid for the rooftop release case. As mentioned earlier, the basic grid had 557,032 cells, where each building side was assigned 20 cells. The coarser grid had 200,900 cells and the finer grid had 1,520,289 cells, where each building side was assigned 15 and 27 cells, respectively. In the results, though a certain deviation was observed between the coarser grid and the basic grid in terms of the peak of the concentration, significant grid sensitivity was not found for the other parts. Therefore, the resolutions of the building and the gas exit adopted in the basic grid were retained for other cases.

\subsection{Boundary conditions}

The boundary conditions were set using basic guidelines (Franke et al., 2007, 2011; Tominaga et al., 2008b), and the profiles of the streamwise velocity and turbulent kinetic energy $k$ were imposed on the inlet based on the experimental data (cf. Fig. 2). The values of $\varepsilon$ were determined based on the assumption of the local equilibrium $P_{k}=\varepsilon$, where $P_{k}$ is the production term in the $k$ equation. The wall function was applied to the ground and building surfaces. The standard wall functions (Launder and Spalding, 1974) were applied to the wall boundaries. The functions were modified to reflect the effect of the roughness on the ground using the equivalent sand-grain roughness height $k_{s}$ and
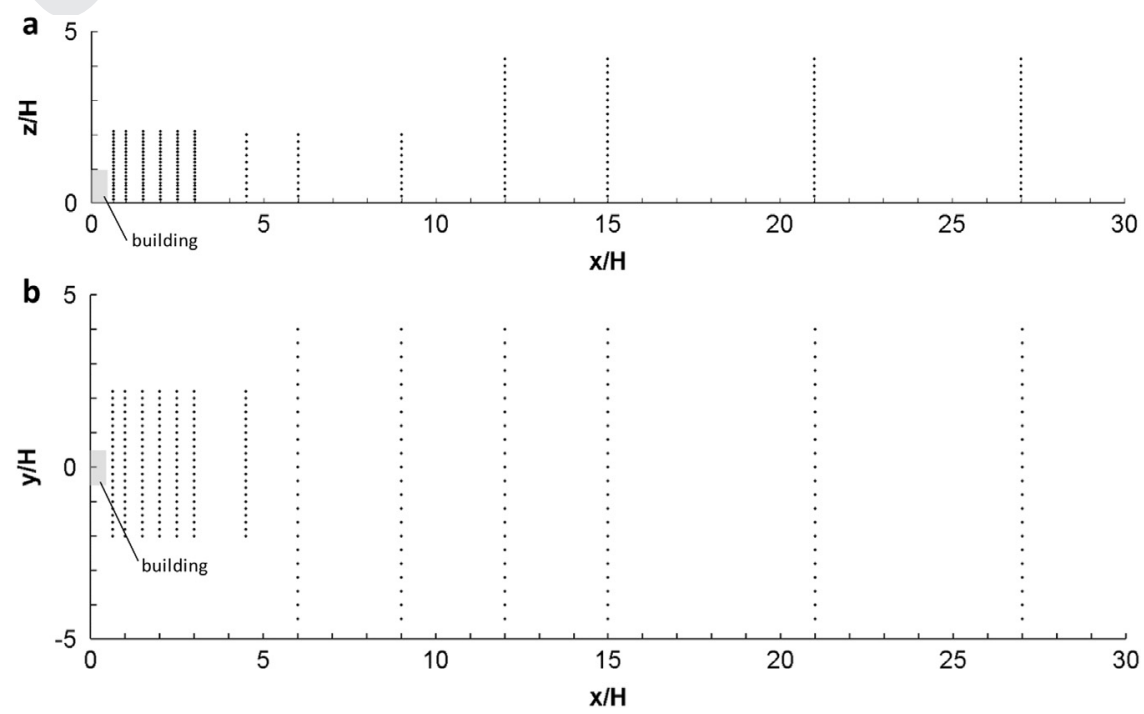

Fig. 4. Measurement points in (a) the vertical mid-plane and $(b)$ the horizontal plane on the ground $(z / H=0)$ for the rooftop release case. 


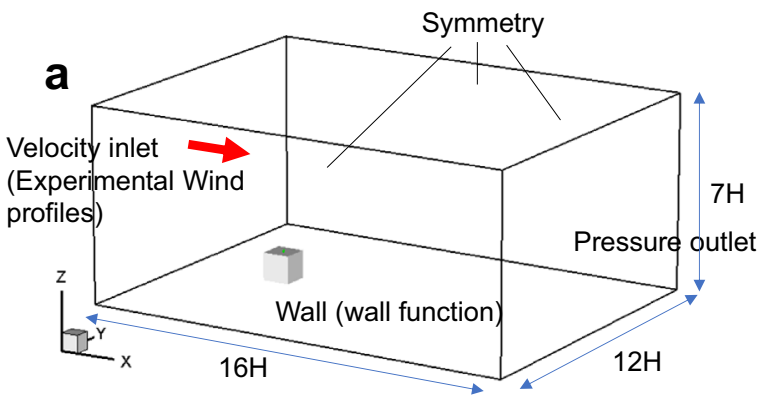

b

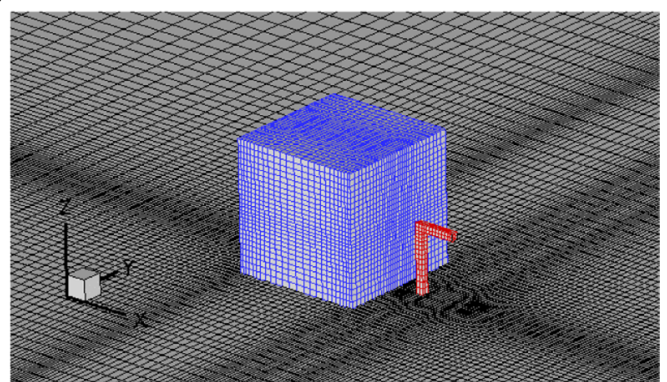

Fig. 5. Computational details: (a) Computational domain, boundary conditions; (b) Computational grid on building and ground surfaces for downwind release case.

roughness constant $C_{s}$. The horizontal inhomogeneity of the atmospheric boundary layer was limited by adapting $k_{s}$ and $C_{s}$ to the inlet profiles using the equation proposed by Blocken et al. (2007), namely, $k_{s}=9.793 z_{0} / C_{s}$, where $z_{0}$ is the aerodynamic roughness length of the terrain. The selected values were $k_{s}=0.6 \times 10^{-5} \mathrm{~m}$ and $C_{s}=1.0$. Symmetric boundary conditions were imposed at the sides and top of the domain, implying zero normal velocity and zero gradients for all variables at these boundaries. Zero static pressure was imposed at the outlet of the domain.

\subsection{Turbulence model}

The Renormalisation Group (RNG) $k-\varepsilon$ model (Yakhot et al., 1992) was adopted, with a modification of the dissipation-rate $\varepsilon$ equation to reflect the lag between the input of energy at a discrete frequency and the response of the dissipative motions, according to Younis and Zhou (2006). The modification was made by introducing an additional term representing a positive (production) source in the $\varepsilon$ equation. The modified production term of $\varepsilon$ is obtained as follows:

$P_{\varepsilon}=C_{\varepsilon 1} P_{k}\left(\frac{1}{T_{t}}+C_{t} \frac{1}{T_{p}}\right)$

$T_{\mathrm{t}}$ in a conventional turbulence closure is usually obtained as $k / \varepsilon$, and $T_{\mathrm{p}}$ is an introduced time scale for the periodic motion. $C_{\mathrm{t}}$ is a proportionality coefficient, whose value here of 0.38 was proposed in the literature (Younis and Zhou, 2006). If the periodic motion is negligible (i.e., $T_{p}=\infty$ ), Eq. (1) would yield the conventional production term of the $\varepsilon$-equation for typical $k-\varepsilon$ models. $T_{p}$ is expressed as follows:

$T_{p}=\frac{Q+k_{t}}{\left|D\left(Q+k_{t}\right) / D t\right|}$

Here, $Q$ is the ensemble mean kinetic energy $\left(\equiv \frac{1}{2} U_{i}^{2}\right)$ and $k_{t}$ is the turbulent kinetic energy. The term $D\left(Q+k_{t}\right) / D t$ can be simplified to $\partial\left(Q+k_{t}\right) / \partial t$ using a fixed grid.

This modification can be applied to any type of $k-\varepsilon$ model. Tominaga (2015) demonstrated that the URANS computation using the RNG $k-\varepsilon$ model could reproduce the periodic fluctuation behind the building by introducing this modification, while improving the prediction accuracy of the velocity field compared to the results of the SRANS computations using the same model for the flow field around a high-rise building with a 1:1:2 shape. The RNG $k-\varepsilon$ model was adopted as a representative of the revised k- $\varepsilon$ models, because among the SRANS computations with five different turbulence models, namely, the standard $k-\varepsilon$ model (Launder and Spalding, 1972), RNG $k-\varepsilon$ model, realizable $k-\varepsilon$ model (Shih et al., 1995), standard $k-\omega$ model (Wilcox, 1998), and the $k-\omega$ shear stress transport (SST) model (Menter, 1994), it showed the best performance in reproducing the reverse flow on the roof. Although the URANS computation using the $k-\omega$ SST model successfully reproduced unsteady fluctuations without this modification, the computation significantly underestimated the turbulent kinetic energy around the building, and the flow separation around the building corners was therefore significantly overestimated. Based on the information from Tominaga (2015), the $\varepsilon$ modification was applied to the RNG $k-\varepsilon$ model in this study.

\subsection{Solver settings}

The commercial software ANSYS FLUENT 14.5 was used for the SRANS and URANS computations. A control volume approach was used to solve the flow equations. All the transport equations were discretized using the QUICK scheme, and the pressure interpolation was of the second order. The semi-implicit method for pressure-linked equations (SIMPLE) algorithm was used for the pressure-velocity coupling of the SRANS computation. The time integration of the URANS computation was second-order implicit, and the non-iterative fractional step method, with the time step set to $1 \times 10^{-2} \mathrm{~s}$, was used for time advancement. Time-averaging was considered for all statistical values in the URANS for the nondimensional time scale $t^{*}=t U_{H} / H=200$. The performance of this approach was evaluated for the flow field around a high-rise building model (Tominaga, 2015). The local mass fraction of ethane was predicted through the solution of its convection-diffusion equations. The turbulent Schmidt number was set to 0.7 (Tominaga and Stathopoulos, 2007).

\section{SRANS and URANS: Comparison of flow fields}

The flow fields obtained using SRANS and URANS were compared for the condition without gas emissions. The present URANS computation successfully reproduced the periodic fluctuation around the building. Fig. 6 shows time histories of the lateral velocity $\mathrm{V}$ at the three different source positions. Note that points $\mathrm{A}$ and $C$ exactly correspond to the source locations for the upwind and downwind release cases, but point B is located at $0.05 \mathrm{H}$ above on the gas exit for the rooftop release case. A clear periodic fluctuation with a period of $t^{*} \approx 12$ is evident. The fluctuation amplitude at point $A$ at the front of the building is rather small. However, the amplitudes of the fluctuations at points $B$ and $C$ are relatively large: $0.08 U_{H}$ at point $\mathrm{B}$, located near the roof, and $0.1 U_{H}$ at point $C$, located behind the building. At points $B$ and $C$, the amplitudes are almost identical, but the phases are slightly shifted. The non-dimensional standard deviation of the fluctuations of the lateral velocity $V, \sigma_{v} / U_{H}$, reproduced by URANS is 0.06 at point $C$, whereas it was 0.16 in the experiment at the same point. Even though the turbulent component of the fluctuations is not included in $\sigma_{\mathrm{v}} / \mathrm{U}_{\mathrm{H}}$ by URANS, it seems to be much weaker than that observed in the experiment (Tominaga, 2015).

The mean streamlines and total turbulent kinetic energy $k_{\text {total }}$ at the vertical center section are shown in Fig. 7 . $k_{\text {total }}$ can be calculated by summing its two components, namely, the turbulent 


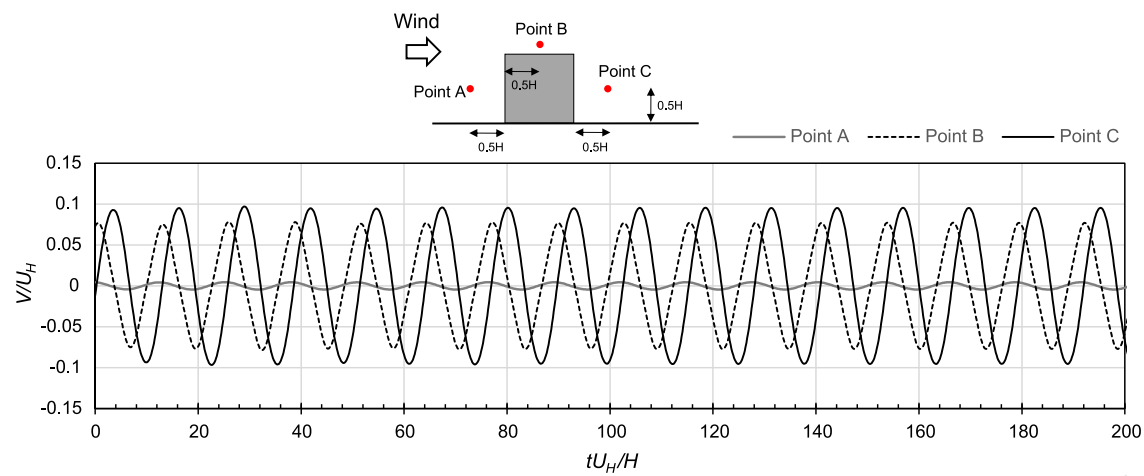

Fig. 6. Time histories of the ensemble-averaged lateral velocity $V$ at (near) the source position obtained by URANS computation without gas emission.
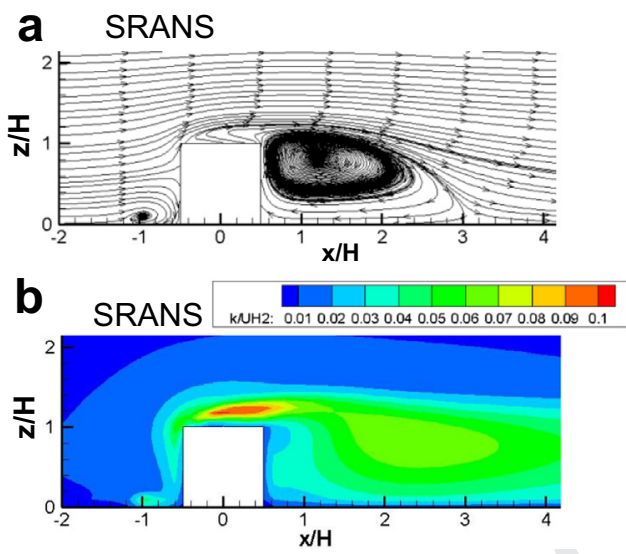
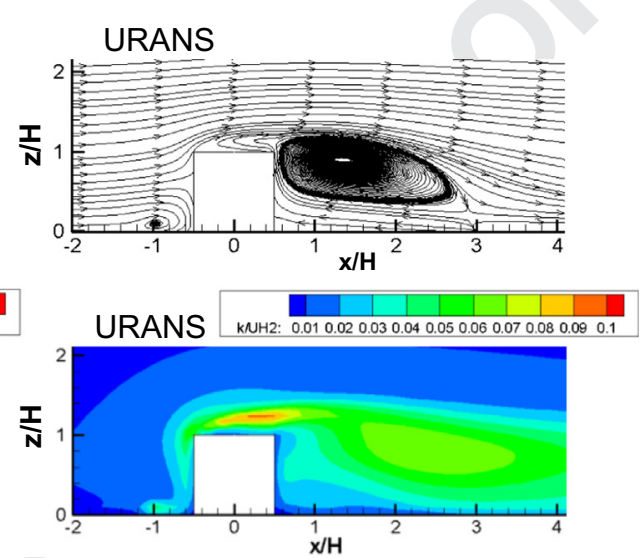

Fig. 7. Comparison of flow fields for SRANS and URANS: (a) Streamlines of mean flow, (b) Total turbulent kinetic energy $k_{\text {total }}$.

kinetic energy due to the stochastic turbulent fluctuation $k_{\mathrm{t}}$, and that due to the periodic fluctuation produced by the large-scale flow, $k_{\mathrm{p}}$.

$k_{\text {total }}=k_{t}+k_{p}$

$$
k_{p}=\frac{1}{2} \overline{\tilde{u}_{i}^{2}}
$$

$\tilde{\boldsymbol{u}}_{\boldsymbol{i}}$ : periodic fluctuations, $\tilde{\boldsymbol{u}}_{\boldsymbol{i}}=\boldsymbol{U}_{\boldsymbol{i}}-\overline{\boldsymbol{u}}_{\boldsymbol{i}}$

$\boldsymbol{U}_{i}$ : ensemble-averaged velocity

$\overline{\boldsymbol{u}}_{\boldsymbol{i}}$ : time-averaged velocity

Here, $k_{\mathrm{t}}$ can be obtained by simply solving its transport equation using the RANS computation, whereas $k_{\mathrm{p}}$ is calculated by the time integration of the unsteady fluctuation of the ensembleaveraged velocity $U_{\mathrm{i}}$ over a certain period. Therefore, $k_{\mathrm{total}}=k_{\mathrm{t}}$ for the SRANS computation, and $k_{\text {total }}=k_{\mathrm{t}}+k_{\mathrm{p}}$ for the URANS computation. Fig. 8 compares the vertical and horizontal distributions of the streamwise component of the time-averaged velocity $U$ and $k_{\text {total }}$ obtained by SRANS and URANS computations with respective experimental values. The agreement between the experimental and computational results is generally good. The differences between the SRANS and URANS results are small in the present case, in contrast to those in previous studies in which SRANS and URANS were compared (Iaccarino et al., 2003; Tominaga, 2015). Fig. 9 compares the two components of total kinetic energy, i.e., the stochastic turbulent component, $k_{t}$ (SRANS and URANS) and the periodic component, $k_{p}$ (only URANS) at the vertical center plane. The URANS computation slightly underestimates the stochastic turbulent component, $k_{t}$, in comparison with the SRANS, because the effect of the $\varepsilon$ modification acts as a positive source term in the $\varepsilon$ equation. The periodic component, $k_{p}$, in URANS is relatively small compared to the stochastic component, $k_{t}$. Therefore, because the increase in $k_{p}$ is offset by the decrease in $k_{t}$ in URANS, the difference in the total turbulent kinetic energy $\left(k_{p}+k_{t}\right)$ between SRANS and URANS is small in the present study.

\section{SRANS and URANS: Comparison of concentration fields}

All concentrations are expressed in this paper in non-dimensional form. The non-dimensional concentration $C^{*}$ is defined as follows:

$C^{*}=\frac{C}{C_{0}}$

where $C_{0}$ is the reference concentration, expressed as:

$C_{0}=\frac{Q_{e}}{H^{2} U_{H}}$

and $Q_{e}$ is the pollutant exhaust rate.

The following validation metrics were used to quantify the agreement between the computational and experimental results (Schatzmann et al., 2009): the hit rate $q$, the fraction of the prediction within a factor of 2 of the observations (FAC2), the fractional bias (FB), and the normalized mean square error (NMSE). These metrics can be expressed as follows:

$q=\frac{1}{N} \sum_{i=1}^{N} n_{i}$ with $n_{i}=\left\{\begin{array}{l}1 \text { for }\left|\frac{P_{i}-O_{i}}{O_{i}}\right| \leq D_{q} \text { or }\left|P_{i}-O_{i}\right| \leq W_{q} \\ 0 \text { else }\end{array}\right.$

$F B=\frac{[O]-[P]}{0.5([O]+[P])}$ 

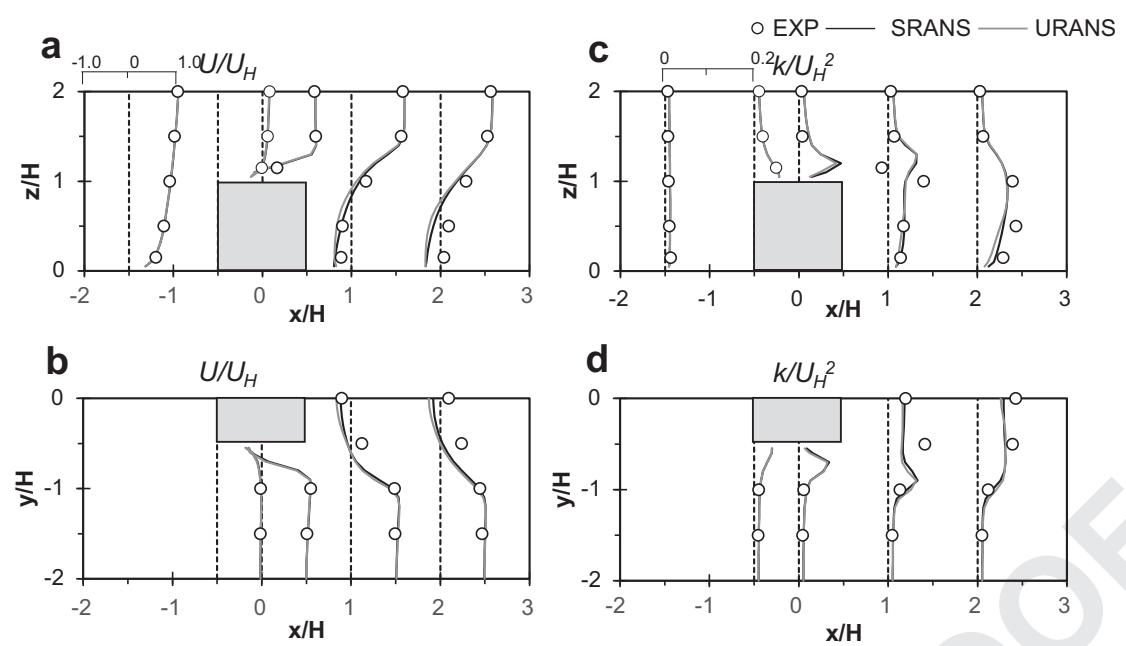

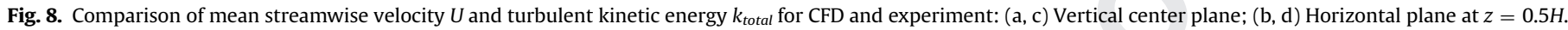

\section{a}

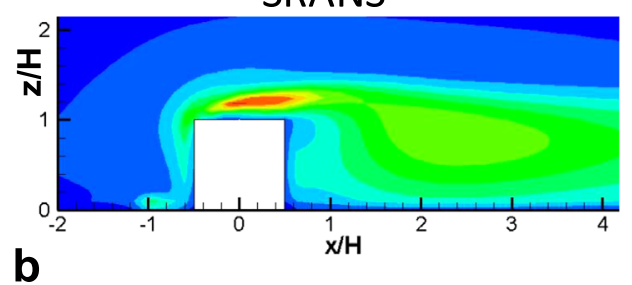

not reproduced
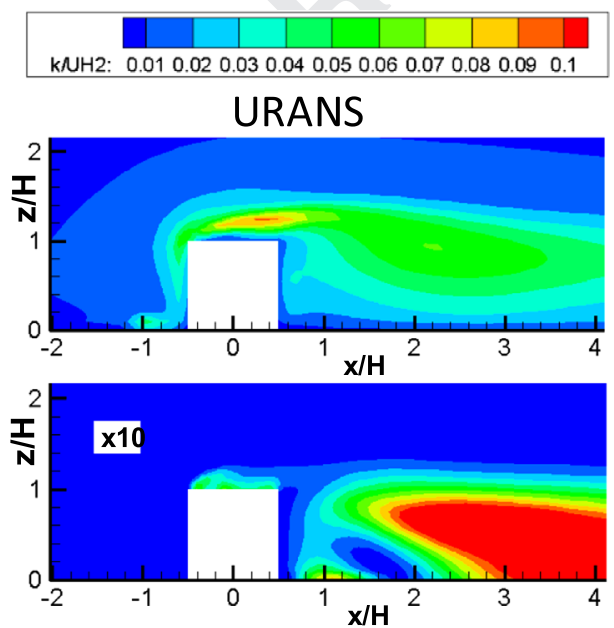

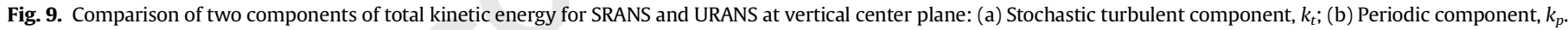

NMSE $=\frac{\left[\left(O_{i}-P_{i}\right)^{2}\right]}{[O][P]}$

$F A C=\frac{1}{N} \sum_{i=1}^{N} n_{i}$ with $n_{i}=\left\{\begin{array}{l}1 \text { for } 0.5 \leq \frac{P_{i}}{O_{i}} \leq 2 \\ 0 \text { else }\end{array}\right.$

In these definitions, $O_{\mathrm{i}}$ and $P_{\mathrm{i}}$ are the observed (measured) and predicted (computed) values of a given variable for sample i, respectively, and $N$ is the number of data points. The square brackets denote averaging over the entire dataset. The ideal values of the metrics that correspond to perfect agreement are 1.0 for $q$ and FAC2 and 0 for FB and NMSE. Previous studies suggest the following judgment criteria for these metrics for concentration: FAC2 $>0.5,|F B|<0.3$, NMSE $<4$ (Schatzmann et al., 2009; Hanna et al., 2004).

In this study, the metrics for mean concentration consider 216, 187 and 143 measurement points on the vertical plane $(y=0)$ and 108,117 and 93 points on the horizontal plane $(z=0)$ for the upwind, rooftop and downwind release cases, respectively. The thresholds for $q$ are $D_{\mathrm{q}}=0.25$ and $W_{\mathrm{q}}=0.05$ (Schatzmann et al., 2009). The computational results of the metrics for SRANS and URANS are listed in Table 1.
Table 1

Validation metrics for the three cases considered in the present study.

\begin{tabular}{|c|c|c|c|c|c|c|c|c|}
\hline \multirow[t]{2}{*}{ Cases } & \multicolumn{4}{|c|}{ SRANS } & \multicolumn{4}{|c|}{ URANS } \\
\hline & $q$ & FAC2 & FB & NMSE & $q$ & FAC2 & FB & NMSE \\
\hline Upwind release & 0.24 & 0.70 & 0.06 & 2.56 & 0.27 & 0.63 & 0.09 & 2.13 \\
\hline Rooftop release & 0.55 & 0.76 & -0.28 & 0.84 & 0.60 & 0.81 & -0.14 & 0.82 \\
\hline Downwind release & 0.44 & 0.84 & -0.43 & 1.25 & 0.65 & 0.83 & 0.03 & 0.83 \\
\hline
\end{tabular}

\subsection{Upwind release case}

Table 1 shows that there is no significant difference between SRANS and URANS. The value of NMSE, which expresses the variability of data, is slightly improved in URANS. This is because the overestimation of concentration, observed in both computations, is slightly improved in URANS as indicated in the scatter plot of Figs. 10 and 11 shows the comparison of the mean non-dimensional concentration $C^{*}$ for SRANS and URANS at the vertical center plane $(y=0)$ and the horizontal plane on the ground $(z=$ $0)$. In both CFD computations, the concentrations behind the building are underestimated, as shown in Fig. 11(a). At the horizontal plane - see Fig. 11(b) - a peak of the concentration is observed at the side of the building in both CFD cases; its locations 

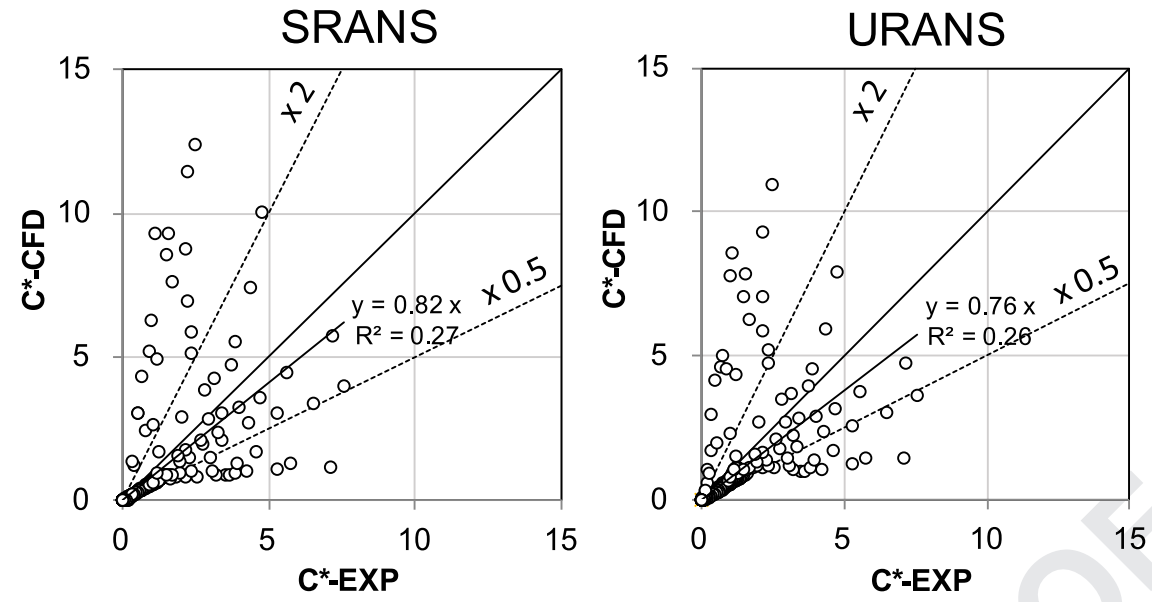

Fig. 10. Scatter plots of non-dimensional concentration $C^{*}$ for upwind release case for SRANS and URANS in comparison with respective experimental values.
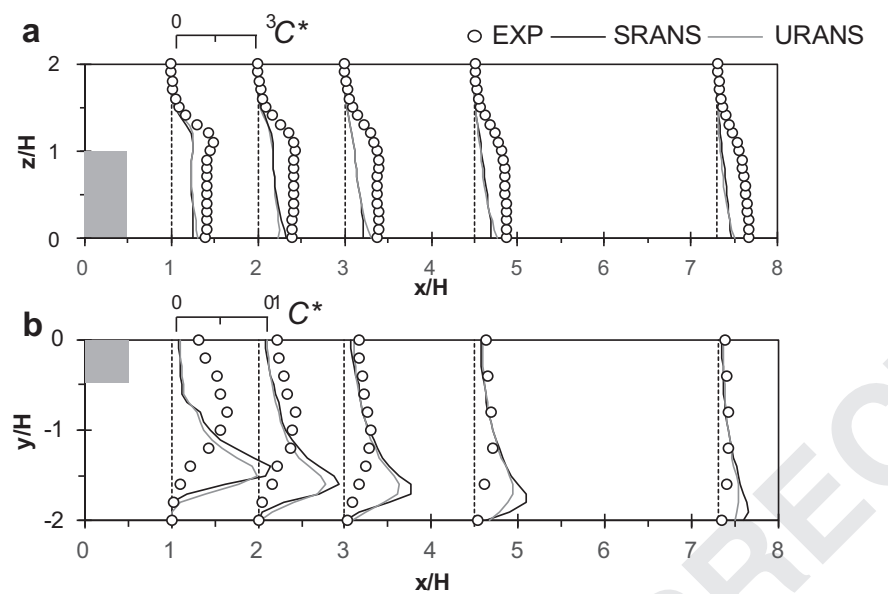

Fig. 11. Comparison of mean non-dimensional concentration $C^{*}$ for SRANS and URANS; (a) vertical center plane $y=0$, (b) horizontal plane $z=0$ (on the ground).

differ from that of the experiment. Although this peak value becomes slightly smaller in URANS than SRANS, the value is still overestimated. This is because the concentration released at the upwind position was more resistant to entrainment to the wake region of the building in CFD than it was in the experiment. Fig. 12 shows the iso-surfaces of the mean non-dimensional concentration $C^{*}=10$ around the building obtained by the SRANS and URANS computations. It is clearly observed that the concentrations are transported to the downwind direction by the horseshoe vortex in front of the building. However, the iso-surfaces of the concentrations are straightly extended to the downwind direction with that of SRANS going deeper in comparison with that of

URANS. This can be explained based on the temporal velocity fluctuation at the source location that is very small, as shown in Fig. 6.

\subsection{Rooftop release case}

Table 1 shows clearly that the general performance of CFD predictions for the rooftop release case is the best among the three cases. In this case, the shortcoming of RANS is not apparent, although URANS computation slightly improves all metrics relative to SRANS. Fig. 13 shows the scatter plots of the non-dimensional concentration $C^{*}$ by SRANS and URANS in comparison with experimental results. While SRANS shows a rather high concentration relative to the experiment, URANS predicts a concentration generally lower than that of SRANS and shows a gradient close to one in the approximate expression. Fig. 14 compares the mean non-dimensional concentration $C^{*}$ for SRANS and URANS at the vertical center plane $(y=0)$ and the horizontal plane on the ground $(z=0)$. In the vertical section, both CFD computations estimate the concentration peak at the building height to be larger than experimentally measured. However, the overestimation is slightly improved by URANS. On the ground, the lateral gradient of the concentration becomes gentle in URANS and the concentration distribution by the experiment is well reproduced, especially at $x /$ $\mathrm{H}=6.0$. Fig. 15 shows the iso-surfaces of the mean non-dimensional concentration $C^{*}=1$ around the building obtained by the SRANS and URANS computations. Although there is no large difference, the shape predicted by SRANS is developed more sharply in the downwind direction than that of URANS. The more diffusive concentration distributions in URANS are attributed to the reproduction of large-scale velocity fluctuations near the source and behind the building, as shown in Fig. 6 .

SRANS

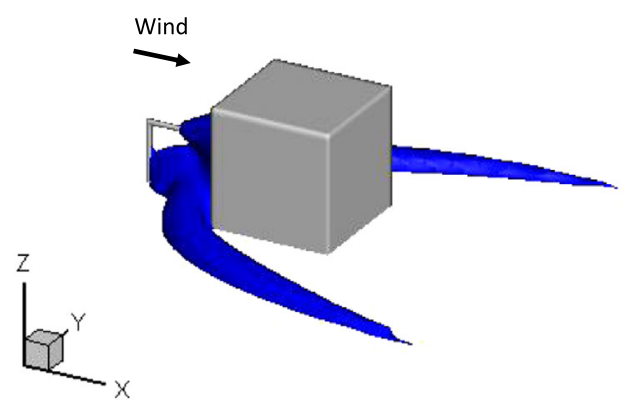

URANS

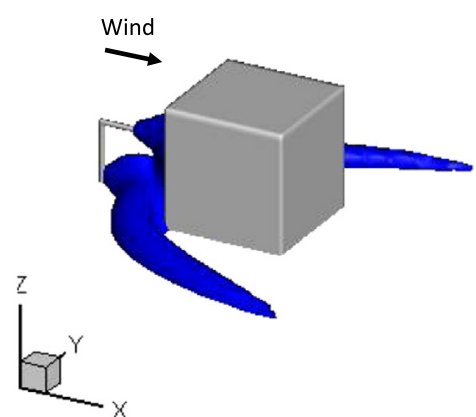



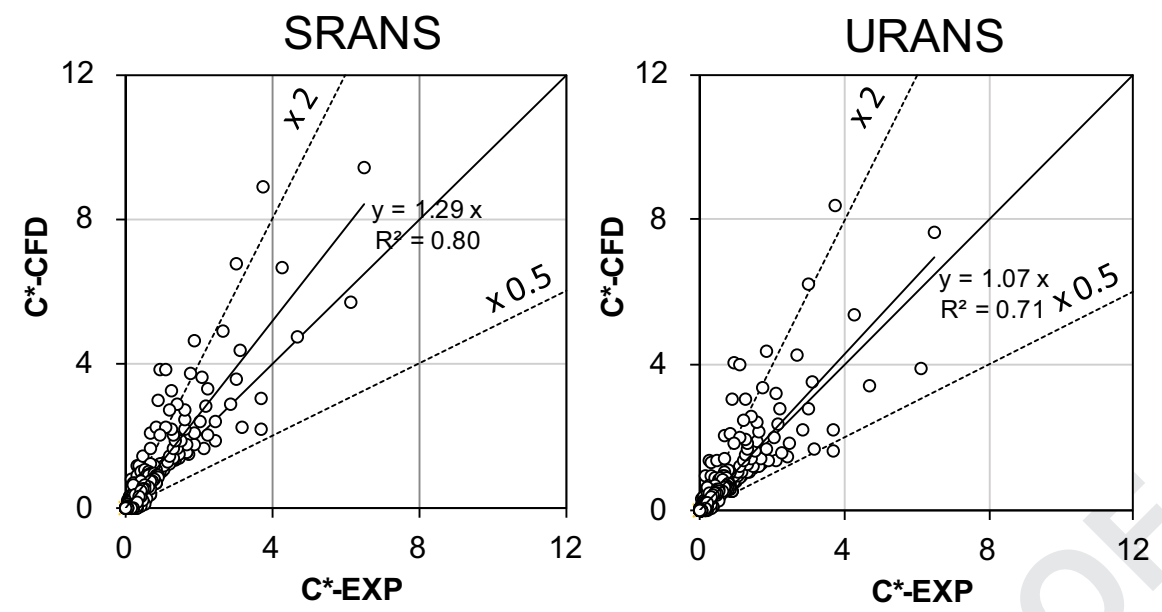

Fig. 13. Scatter plots of non-dimensional concentration $C^{*}$ for rooftop release case using SRANS and URANS in comparison with respective experimental results.
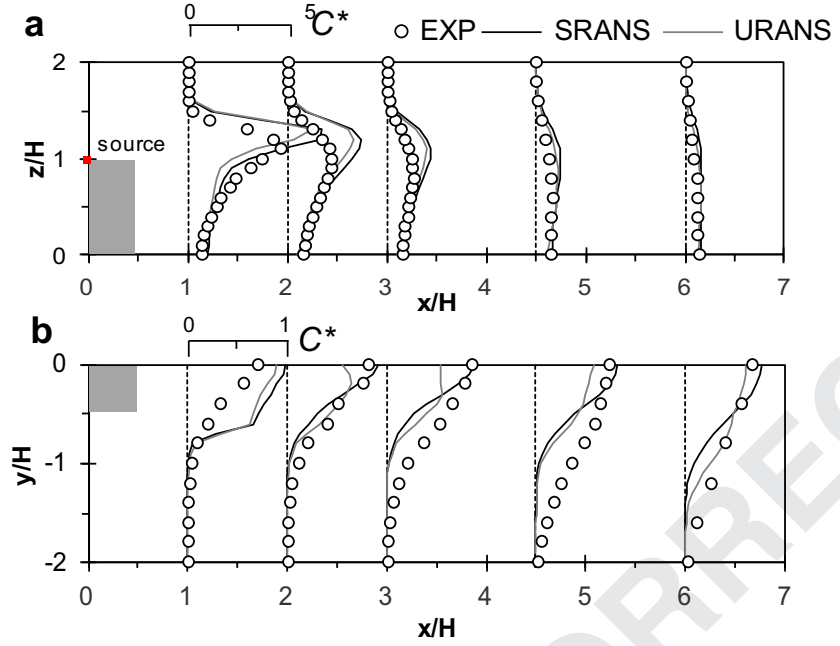

Fig. 14. Comparison of mean non-dimensional concentration $C^{*}$ for rooftop release case using SRANS and URANS: (a) vertical center plane $y=0$, (b) horizontal plane $z$ $=0$ (on the ground).

\subsection{Downwind release case}

Table 1 shows that the performance of URANS is most improved over that of SRANS for the downwind release case. This is probably because the largest velocity fluctuation is reproduced by URANS at the source location (see Fig. 6). Scatter plots of the nondimensional concentrations $C^{*}$ by SRANS and URANS in comparison with those measured in the experiment are shown in Fig. 16. Although no large differences are seen in the values of FAC2, many points are concentrated around the $45^{\circ}$ line in the URANS case. Fig. 17 compares the distributions of the non-dimensional concentration $C^{*}$ behind the building. For the vertical distribution shown in Fig. 17(a), the concentration near the source is largely overestimated by the SRANS computation. However, this overestimation is improved in URANS. Fig. 17(b) shows the ground plane URANS results, in which the concentration diffusion in the lateral direction is estimated to be larger than that in the SRANS results and agrees well with the experiment. Fig. 18 shows the isosurfaces of the mean non-dimensional concentration $C^{*}=$ 1 around the building obtained by the SRANS and URANS computations. The difference between SRANS and URANS is the greatest for this configuration. The shape is stretched sharply in the downwind direction in SRANS, but is shorter and more rounded in URANS. The effect of the time-dependent organized motion reproduced by URANS on the concentration field, the time-series iso-surfaces of the ensemble-averaged non-dimensional concentration $C^{*}=1$ around the building is illustrated in Fig. 19. The time-dependent periodic fluctuation of the ensemble-averaged concentration is clearly observed.

\section{Additional considerations}

The performance of URANS simulations for flow and dispersion fields around isolated cubical buildings was examined by comparing the results with those obtained from SRANS computations and experiments. The following points should be considered in the comparisons:

- The present URANS computations, which used the RNG $k-\varepsilon$ model with the $\varepsilon$ modification, could reproduce the periodic fluctuation around the building. However, the $\varepsilon$ modification is not the only way to reproduce the fluctuation. In fact, the $k-\omega$ SST model could also reproduce the periodic fluctuation around

\section{SRANS}

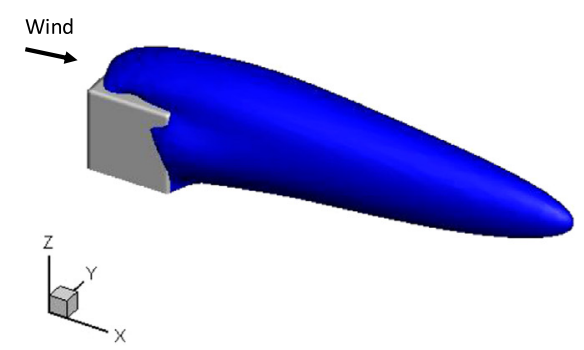

URANS

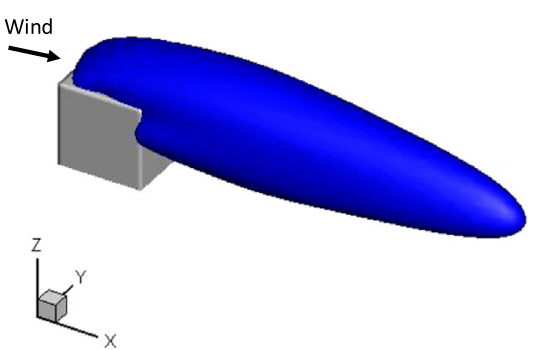



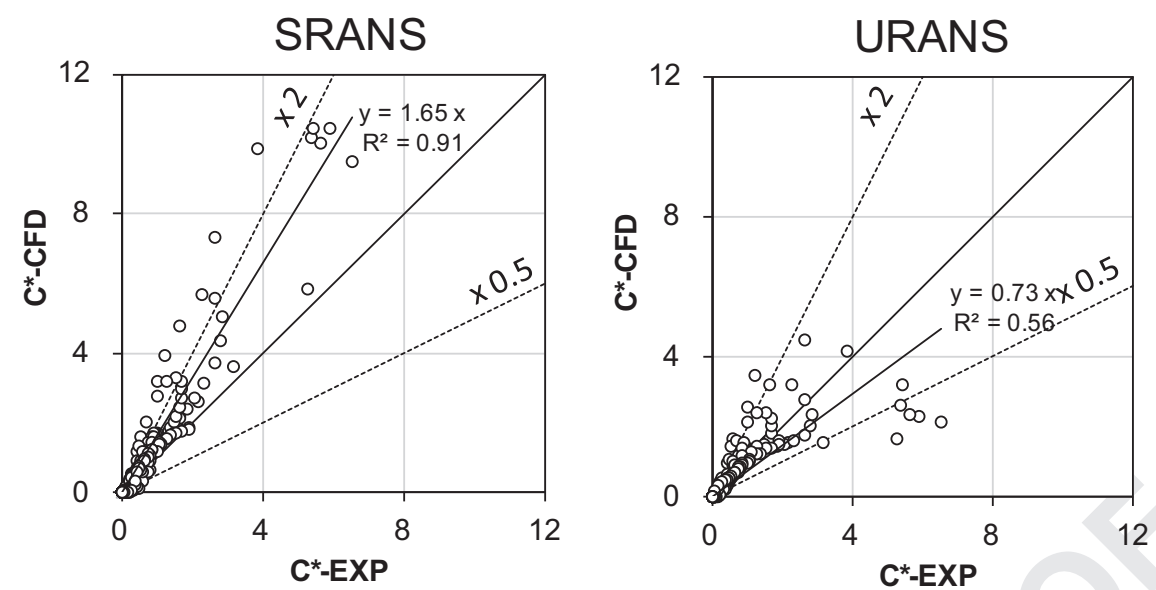

Fig. 16. Scatter plots of non-dimensional concentration for downwind release case using SRANS and URANS in comparison with respective experimental results.
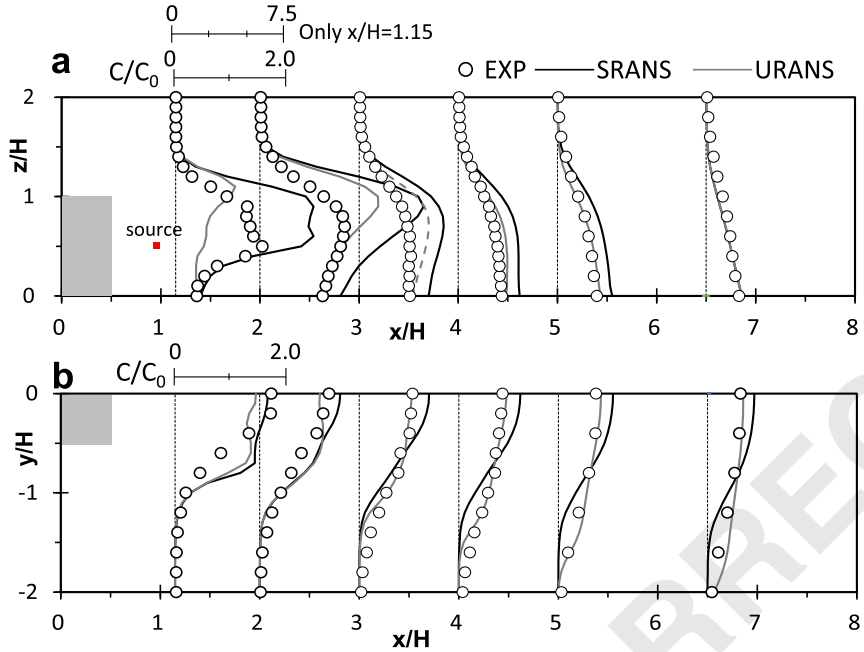

Fig. 17. Comparison of mean non-dimensional concentration $C^{*}$ for downwind release case using SRANS and URANS: (a) vertical center plane $y=0$, (b) horizontal plane $z=0$ (on the ground).

the building under the same computational conditions of the present study in our preliminary tests. The present study used the same turbulence model in SRANS and URANS, namely the turbulence model that showed good performance in the SRANS computation in a previous study (Tominaga, 2015). The combination of URANS and other turbulence models needs more consideration in further study.

- Fig. 6 shows that the large-scale fluctuations reproduced by URANS were quite monotonic. Since the fluctuations with other frequencies were not reproduced, there was probably a gap

between the energy of velocity fluctuations in URANS simulations and the actual total energy of those in reality. This gap may be a limitation of URANS.

- The run time required by the URANS computation was approximately five times longer than that by SRANS for each case. These differences were simply caused by the computational time required by time-averaging for a statistically long enough period. Although it is difficult to directly compare the computational time of RANS with that of LES, the run time of LES can be estimated to be approximately ten times longer than that of URANS. This estimation is based on the assumption that the number of grids required will be about $3.3\left(=1.5^{3}\right)$ times more and the time-averaging period will be about three times longer in LES than those required in URANS.

- For a flow around an isolated building, the large-scale organized motions with periodic flows, such as that observed in the present study, are relatively prominent. However, in a flow around a group of buildings, such flows may disappear into the large turbulence generated by the buildings. A more realistic case with non-isolated buildings should be considered in order to investigate the effect of surroundings on the performance of URANS. In fact, Paik et al. (2009) investigated the performance of URANS computation and various versions of detached eddy simulation (DES) in resolving coherent structures in turbulent flow around two cubes mounted in tandem on a flat plate for a thin incoming boundary layer. They concluded that URANS failed to capture key features of the mean flow, including the second horseshoe vortex in the upstream junction, and significantly underestimated turbulence statistics in most regions of the flow. Salim et al. (2011) compared the performance between SRANS, URANS and LES in the prediction of airflow and pollutant dispersion within urban street canyons. They reported

SRANS

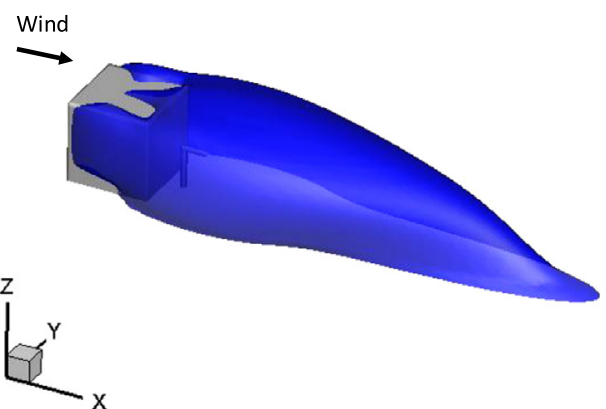

URANS
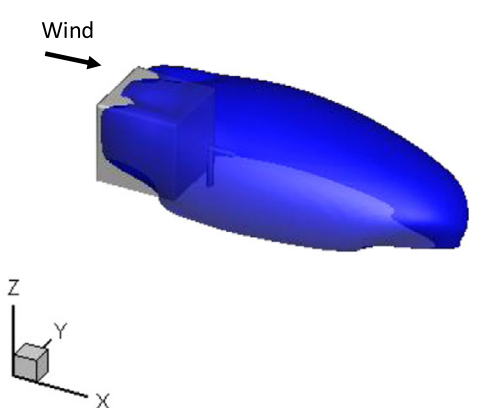

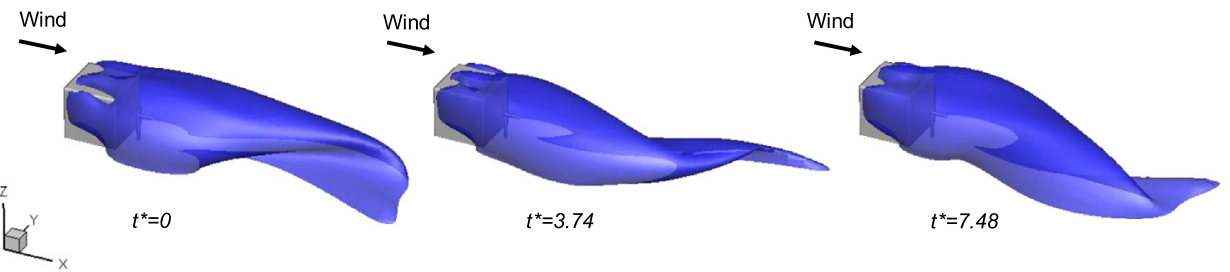

Fig. 19. Time series of the iso-surfaces of the ensemble-averaged non-dimensional concentration $C^{*}=1$ for downwind release case obtained by URANS computation

that although solving for a transient solution, the results for URANS did not vary with time when compared with those of LES.

\section{Conclusions}

The performance of URANS simulations of flow and dispersion fields around isolated cubical buildings with different pollutant release points was examined by comparing the results with those obtained by SRANS simulations and experiments.

The following conclusions can be drawn from the results of this research study:

- The recirculation flow behind the building was estimated by URANS to be slightly shorter than that by SRANS. However, the differences between SRANS and URANS were found to be small for this case, in contrast to the results of previous studies that compared SRANS and URANS. This is attributed to the increase in the periodic component of $k$ being offset by the decrease in the turbulent component of $k$ in URANS in the present study.

- It should be emphasized that the prediction accuracy of CFD depends primarily on the source location for both SRANS and URANS. In this study, both computational approaches yielded better predictions for the rooftop release case than for the other release cases.

- The improvement of the predicted concentration field achieved by URANS largely depends on the source location. Although this improvement was not as large for the upwind and rooftop release cases, the prediction accuracy achieved by URANS was substantially better for the downwind release case.

- For the upwind release case, the effect of unsteady fluctuations is very small, but the overestimated concentration in SRANS is slightly decreased in URANS.

- For the rooftop release case, the prediction accuracy for the lowconcentration region in the downwind region was improved in URANS. The more diffusive concentration distributions in URANS were attributed to the reproduction of large-scale velocity fluctuations near the source and behind the building.

- For the downwind release case, the concentration diffusion in the lateral direction behind the building was estimated to be larger in URANS, and the concentration distribution agrees very well with the results of the experiment.

- This study evaluated the contribution of unsteady large-scale fluctuations to the pollutant dispersion around an isolated building. Such contribution should be carefully considered in validation studies using SRANS for pollutant dispersion around an isolated building, which is often adopted in such studies.

\section{Acknowledgements}

The authors would like to express their gratitude for the financial support provided to the first author through the Grants-inAid for Scientific Research in Japan (No. 25420616) and that to the second author through the Discovery Grant program of the Natural Sciences and Engineering Research Council of Canada (NSERC). The authors are grateful to Professor Robert N. Meroney, member of the Journal's Editorial Board, who kindly agreed to be Acting Editor and handled the review process for this paper outside the EVISE system, since the Editor is a co-author. Finally, the authors would also like to thank Mr. Yuta Kato, an undergraduate student at the Niigata Institute of Technology, for his invaluable assistance with the numerical simulations.

\section{References}

AIST, 2013. Wind tunnel experiments for validation of the DiMCFD model, 〈https:// unit.aist.go.jp/emri/ja/results/db/01/db_01.html $\rangle$ (accessed on 26 Oct. 2016) [in Japanese].

Bechmann, A., 2006. Large-eddy simulation of atmospheric flow over complex terrain. Riso National Laboratory, Tech. Univ. of Denmark, Report Riso-PhD-28 (EN), Chapter 8: Simulation of Wall Mounted Cube, 106 pp.

Blocken, B., Stathopoulos, T., Carmeliet, J., 2007. CFD simulation of the atmospheric boundary layer: wall function problems. Atmos. Environ. 41, 238-252.

Blocken, B., Stathopoulos, T., Carmeliet, J., Hensen, J.L.M., 2011. Application of CFD in building performance simulation for the outdoor environment: an overview. J. Build. Perform. Simul. 4, 157-184.

Blocken, B., 2014. 50 years of computational wind engineering: past, present and future. J. Wind Eng. Ind. Aerodyn. 129, 69-102.

Dejoan, A., Santiago, J.L., Martilli, A., Martin, F., Pinelli, A., 2010. Comparison between large-eddy simulation and Reynolds-averaged Navier-Stokes computations for the MUST field experiment. Part II: effects of incident wind angle deviation on the mean flow and plume dispersion. Bound.-Layer Meteorol. 135, 133-150.

Di Sabatino, S., Buccolieri, R., Salizzoni, P., 2013. Recent advancements in numerical modelling of flow and dispersion in urban areas: a short review. Int. J. Environ. Pollut. 52 (3/4), 172-191.

Durbin, P.A., 1995. Separated flow computations with the $\mathrm{k}-\varepsilon-\mathrm{v}^{2}$ model. AIAA J. 33 , 659-664.

Franke, J., Hellsten, A., Schlünzen, H., and Carissimo, B., 2007. Best Practice Guideline for the CFD Simulation of Flows in the Urban Environment. COST Office, ISBN: 3-00-018312-4, Brussels, Belgium.

Franke, J., Hellsten, A., Schlünzen, H., Carissimo, B., 2011. The COST 732 best practice guideline for CFD simulation of flows in the urban environment - a summary. Int. J. Environ. Pollut. 44 (1-4), 419-427.

Gousseau, P., Blocken, B., Stathopoulos, T., van Heijst, G.J.F., 2011a. CFD simulation of near-field pollutant dispersion on a high-resolution grid: a case study by LES and RANS for a building group in downtown Montreal. Atmos. Environ. 45, 428-438.

Gousseau, P., Blocken, B., van Heijst, G.J.F., 2011b. CFD simulation of pollutant dispersion around isolated buildings: on the role of convective and turbulent mass fluxes in the prediction accuracy. J. Hazard. Mater. 194, 422-434.

Hanna, S.R., Hansen, O.R., Dharmavaram, S., 2004. FLACS CFD air quality model performance evaluation with Kit Fox, MUST, Prairie Grass, and EMU observations. Atmos. Environ. 38 (28), 4675-4687.

Iaccarino, G., Ooi, A., Durbin, P.A., Behnia, M., 2003. Reynolds averaged simulation of unsteady separated flow. Int. J. Heat Fluid Flow 24, 147-156.

Iizuka, S., Kondo, H., Muto, K., Hori, A., Kitabayashi, K., Mizuno, T., 2008. A windtunnel study on gaseous diffusion around a building within two different atmospheric boundary layers. In: Proceedings of the 4th International Conference on Advances in Wind and Structures (AWAS'08), Jeju, Korea, May 20-31, 2008.

Isaev, S.A., Lysenko, D.A., 2009. Calculation of unsteady flow past a cube on the wall of a narrow channel using URANS and the Spalart-Allmaras turbulence model. J. Eng. Phys. Thermophys. 82 (3), 488-495.

Kato, M., and Launder, B.E., 1993. The modelling of turbulent flow around stationary and vibrating square cylinders. In: Proceedings of the Ninth Symposium on Turbulent Shear Flows, 10-14, Kyoto, Japan, August 16-18, 1993.

Kondo, H., Horiuchi, K., Hirano, Y., Maeyama, N., Ogata, K., Iizuka, S., Mizuno, T., 2009. Attempt to make guideline to use CFD model for atmospheric environmental assessment in urban area in Japan, In Proceedings of the Seventh International Conference on Urban Climate, Yokohama, Japan, June 29- July 3, 2009. 
Lateb, M., Meroney, R.N., Yataghene, M., Fellouah, H., Saleh, F., Boufadel, M.C., 2016. On the use of numerical modelling for near-field pollutant dispersion in urban environments: a review. Environ. Pollut. 208 (Part A), 271-283.

Launder, B.E., Spalding, D.B., 1972. Mathematical Models of Turbulence. Academic Press, Press, New York.

Launder, B.E., Spalding, D.B., 1974. The numerical computation of turbulent flows. Comput. Methods Appl. Mech. Eng. 3 (2), 269-289.

Li, X.X., Liu, C.H., Leung, D.Y.C., Lam, K.M., 2006. Recent progress in CFD modelling of wind field and pollutant transport in street canyons. Atmos. Environ. 40, $5640-5658$.

Mannini, C., Šoda, A., Schewe, G., 2010a. Unsteady RANS modelling of flow past a rectangular cylinder: Investigation of Reynolds number effects. Comput. Fluids 39 (9), 1609-1624.

Mannini, C., Šoda, A., Voß, R., Schewe, G., 2010b. Unsteady RANS simulations of flow around a bridge section. J. Wind Eng. Ind. Aerodyn. 98 (12), 742-753.

Menter, F.R., 1994. Two-equation eddy-viscosity turbulence models for engineering applications. AIAA J. 32, 1598-1605.

Meroney, R., Ohba, R., Leitl, B., Kondo, H., Grawe, D., Tominaga, Y., 2016. Review of CFD guidelines for dispersion modeling. Fluids 1 (2), 14.

Mizuno, T., (ed.) 2013. Guidelines on atmospheric environmental assessment using CFD models (DiMCFD), Subcommittee on prediction, planning and assessment, Kanto blanch, Japan Society for Atmospheric Environment [in Japanese].

Murakami, S., Mochida, A., Hayashi, Y., Sakamoto, S., 1992. Numerical study on velocity-pressure field and wind forces for bluff bodies by k- $\varepsilon$, ASM and LES. J. Wind Eng. Ind. Aerodyn. 41-44, 2841-2852.

Murakami, S., Mochida, A., 1995. On turbulent vortex shedding flow past 2D square cylinder predicted by CFD. J. Wind Eng. Ind. Aerodyn. 54/55, 191-211.

Paik, J., Sotiropoulos, F., Porte-Agel, F., 2009. Detached-eddy simulation of flow around two wall-mounted cubes in tandem. Int. J. Heat Fluid Flow 30, 286-305.

Pellegrino, A., Meskell, C., 2013. Vortex shedding from a wind turbine blade section at high angles of attack. J. Wind Eng. Ind. Aerodyn. 121, 131-137.

Ramesh, V., Vengadesan, S., Narasimhan, J.L., 2006. 3D unsteady RANS simulation of turbulent flow over bluff body by non-linear model. Int. J. Numer. Methods Heat Fluid Flow 16 (6), 660-673.

Rodi, W., 1993. On the simulation of turbulent flow past bluff bodies. J. Wind Eng. Ind. Aerodyn. 46-47, 3-19.

Rodi, W., 1997. Comparison of LES and RANS calculations of the flow around bluff bodies. J. Wind Eng. Ind. Aerodyn. 69, 55-75.

Salim, S.M., Buccolieri, R., Chan, A., Di Sabatino, S., 2011. Numerical simulations of atmospheric pollutant dispersion in an urban street canyon: comparison between RANS and LES. J. Wind Eng. Ind. Aerodyn. 99, 103-113.

Salim S.M., Ong, K.C., Cheah, S.C., 2011. Comparison of RANS, URANS and LES in the Prediction of Airflow and Pollutant Dispersion. In: Proceedings of the World Congress on Engineering and Computer Science 2011 (WCECS 2011), Vol II, San Francisco, USA, October 19-21, 2011.

Santiago, J.L., Dejoan, A., Martilli, A., Martin, F., Pinelli, A., 2010. Comparison between large-eddy simulation and Reynolds-averaged Navier-Stokes computations for the MUST field experiment. Part I: Study of the flow for an incident wind directed perpendicularly to the front array of containers. Bound.-Layer Meteorol. 135, 109-132.

Schatzmann M., Olesen H., Franke J., (Eds.), 2009. COST 732 Model Evaluation Case Studies: Approach and Results, COST Office Brussels, Belgium.

Shao, J., Liu, J., Zhao, J., 2012. Evaluation of various non-linear $k-\varepsilon$ models for predicting wind flow around an isolated high-rise building within the surface boundary layer. Build. Environ. 57, 145-155.

Shih, T.-H., Liou, W.W., Shabbir, A., Yang, Z., Zhu, J., 1995. A new k- $\varepsilon$ eddy viscosity model for high Reynolds number turbulent flows. Comput. Fluids 24 (3), 227-238.

Shimada, K., Ishihara, T., 2002. Application of a method k- $\varepsilon$ model to the prediction of aerodynamic characteristics of rectangular cross-section cylinders. J. Fluids Struct. 16 (4), 465-485.

Snyder, W.H., Castro, I.P., 2002. The critical Reynolds number for rough-wall boundary layers. J. Wind Eng. Ind. Aerodyn. 90, 41-54.

Stevens, D.E., Chan, S.T., Lee, R.L., 2000. Model validation of flow and dispersion around a cube. Lawrence Livermore National Laboratory, US DOE, Report UCRLID-137241, January 20, 2000.

Tominaga, Y., Stathopoulos, T., 2007. Turbulent Schmidt numbers for CFD analysis with various types of flowfield. Atmos. Environ. 41, 8091-8099.

Tominaga, Y., Mochida, A., Murakami, S., Sawaki, S., 2008a. Comparison of various revised $\mathrm{k}-\varepsilon$ models and LES applied to flow around a high-rise building model with 1:1:2 shape placed within the surface boundary layer. J. Wind Eng. Ind. Aerodyn. 96 (4), 389-411.

Tominaga, Y., Mochida, A., Yoshie, R., Kataoka, H., Nozu, T., Yoshikawa, M., Shirasawa, T., 2008b. AIJ guidelines for practical applications of CFD to pedestrian wind environment around buildings. J. Wind Eng. Ind. Aerodyn. 96, 1749-1761.

Tominaga, Y., Stathopoulos, T., 2010. Numerical simulation of dispersion around an isolated cubic building: Model evaluation of RANS and LES. Build. Environ. 45 , 2231-2239.

Tominaga, Y., Stathopoulos, T., 2011. CFD modeling of pollution dispersion in a street canyon: comparison between LES and RANS. J. Wind Eng. Ind. Aerodyn. 99, 340-348.

Tominaga, Y., Stathopoulos, T., 2012. CFD modeling of pollution dispersion in building array: evaluation of turbulent scalar flux modeling in RANS model using LES results. J. Wind Eng. Ind. Aerodyn. 104-106, 484-491.

Tominaga, Y., Stathopoulos, T., 2013. CFD simulation of near-field pollutant dispersion in the urban environment: a review of current modeling techniques. Atmos. Environ. 79, 716-730.

Tominaga, Y., 2015. Flow around a high-rise building using steady and unsteady RANS CFD: Effect of large-scale fluctuations on the velocity statistics. J. Wind Eng. Ind. Aerodyn. 142, 93-103.

Tominaga, Y., Stathopoulos, T., 2016. Ten questions concerning modeling of nearfield pollutant dispersion in the built environment. Build. Environ. 105, 390-402.

Wilcox, D.C., 1998. Turbulence Modeling for CFD. DCW Industries, Inc., La Canada, California.

Xie, Z.-T., Castro, I.P., 2009. Large-eddy simulation for flow and dispersion in urban streets. Atmos. Environ. 43, 2174-2185.

Yakhot, V., Orszag, S.A., Thangam, S., Gatski, T.B., Speziale, C.G., 1992. Development of turbulence models for shear flows by a double expansion technique. Phys. Fluids A4, 1510-1520.

Yoshie, R., Jiang, G., Shirasawa, T., Chung, J., 2011. CFD simulations of gas dispersion around high-rise building in non-isothermal boundary layer. J. Wind Eng. Ind. Aerodyn. 99 (4), 279-288.

Younis, B.A., Zhou, Y., 2006. Accounting for mean-flow periodicity in turbulence closures. Phys. Fluids 18, 018102. http://dx.doi.org/10.1063/1.2166458. 